\title{
INCORPORANDO HERRAMIENTAS WEB 2.0 EN EL ÁMBITO UNIVERSITARIO. FACEBOOK COMO HERRAMIENTA DE APOYO A LA ENSEÑANZA EN INFORMATICA.
}

\section{INTRODUCING WEB 2.0 TOOLS AT UNIVERSITY LEVEL. FACEBOOK AS A TOOL TO SUPPORT E- LEARNING IN GEOGRAPHY}

\author{
Lic. Jaquelina E. Escalante \\ Prof. Adjunta Introducción a la Computación. Dpto Geografía Fac. de Humanidades - Becaria \\ Investigación Fac. de Ciencias Exactas y Naturales y Agrimensura. UNNE \\ Mgter Sonia I. Mariño \\ Prof. Titular Fac. de Ciencias Exactas y Naturales y Agrimensura. UNNE \\ Lic. Romina Y. Alderete \\ Adscripta Introducción a la Computación. Dpto Geografía Fac. de Humanidades - Fac. de Ciencias \\ Exactas y Naturales y Agrimensura. UNNE
}

\section{Resumen:}

Las Redes Sociales en Internet se han convertido en el método de encuentro de personas más usado. El sistema educativo no permanece ajeno a este fenómeno social que modifica la forma de comunicación entre las personas. El trabajo describe una experiencia en el uso de una red social como herramienta de comunicación en la asignatura Introducción a la Computación, de la carrera de grado Licenciatura y Profesorado en Geografía de la Universidad Nacional del Nordeste. A continuación se alude a la metodología utilizada para la recolección de información. Los resultados obtenidos indican que el mayor logro en el rendimiento académico se obtuvo de los alumnos que aplicaron esta propuesta. Finalmente se esbozan consideraciones y futuras líneas de investigación.

Palabras Claves: Educación, Redes Sociales, Web 2.0, facebook, Geografía.

\begin{abstract}
:
Internet social networks have become the most used method for human encounters. The education system can not remain oblivious to this social phenomenon that is changing the way of communication between people. This paper describes an experience using social networks as a communication tool for the subject "Introducción a la Computación" of the degree career "Licenciatura y Profesorado en Geografía" at the "Universidad Nacional del Nordeste". Following, the methodology used for data collection is mentioned. Results presented demonstrate an improvement in academic performance of students who applied this proposal. Finally, outlines considerations and future research is presented
\end{abstract}

Keywords: Education, Social Networks, Web 2.0, facebook, Geography.

Publicado en formato digital: Lic. Jaquelina E. Escalante, Mgter Sonia I. Mariño y Lic. Romina Y. Alderete . INCORPORANDO HERRAMIENTAS WEB 2.0 EN EL ÁMBITO UNIVERSITARIO. FACEBOOK COMO HERRAMIENTA DE APOYO A LA ENSEÑANZA EN INFORMATICA Revista Geográfica Digital. IGUNNE. Facultad de Humanidades. UNNE. Año 10. No 20. Julio - Diciembre 2013. ISSN 1668-5180 Resistencia, Chaco. 


\section{SUMARIO DEL ARTÍCULO \\ - INTRODUCCIÓN \\ - MATERIALES Y MÉTODOS \\ - RESULTADOS \\ - CONCLUSIÓN \\ - REFERENCIAS CITADAS}

\section{Introducción}

La Universidad como institución debe responder a la filosofía que subyace al predominio de lo digital y la web social de la actualidad y del futuro con planteamientos integrales, puesto que todas sus unidades y servicios son susceptibles de mejora gracias a la aplicación bien fundamentada de las metodologías de la web 2.0 y posteriores. En concreto, las esferas donde mayor impacto se puede esperar son aquellas vinculadas al proceso de enseñanza/aprendizaje y de producción, validación y difusión de conocimientos. Lo expuesto asegura una formación y actualización en las alfabetizaciones y las competencias de alumnos, profesores y personal, que se engloban en el concepto de multialfabetización (especialmente alfabetización en lectoescritura, en TIC e informacional).

En Rodriguez Navarrete (2013), se sintetizan algunos de los propósitos de la educación en un mundo globalizado:

- Propiciar experiencias de aprendizaje que fomenten la investigación, el descubrimiento y la experimentación.

- Utilizar el aprendizaje colaborativo como técnica didáctica.

- Tomar en cuenta la experiencia del alumno para orientar el aprendizaje con el objetivo de que aprenda por sí mismo, de modo, que este sea elaborado por el propio estudiante. Por ello, es importante implementar estrategias donde se presenten la investigación, las discusiones grupales y la resolución de problemas, entre otros.

Se pretende que los procesos de formación, inviten al estudiante a reflexionar sobre su propio proceso de estructuración del conocimiento, al incluir el establecimiento de objetivos de aprendizaje claros, que dan solidez a las metas propuestas para la asignatura, donde se promueve una autoevaluación conjunta y oportuna (entre profesor y estudiantes) y la reestructuración de los elementos que conforman el curso, como las metodologías utilizadas para enseñar, las bases conceptuales (temáticas), analíticas y racionales (estimulando el proceso de aprender a aprender) de la asignatura, las estrategias didácticas y de evaluación, el uso de las tecnologías de la información y comunicación como complemento del proceso, con el fin de percibir mejoras puntuales en pro de la apropiación de las temáticas. Es decir, coincidiendo con Fuentes Cotes (2013), se concluye que el aprendizaje obtenido al finalizar el curso, debe ser significativo para su desarrollo profesional y que de ninguna manera deberá estar desarticulado con su proyecto integral de vida.

El conductismo, el cognoscitivismo y el constructivismo son las tres grandes teorías de aprendizaje que se utilizan en la educación y que se enfocan a lograr un aprendizaje significativo. Según Valerio y Valenzuela (2010) existe una nueva teoría denominada conectivismo, creada por George Siemens, y destacan que el mismo propuso el concepto de conectivismo aplicado al aprendizaje. Las Redes Sociales son el elemento central del conectivismo. Es en las conexiones donde radica la posibilidad del aprendizaje. Han existido desde hace mucho tiempo; sin embargo, con el avance tecnológico, estas han evolucionado hacia las modernas Redes Sociales en línea.

En el mundo contemporáneo, el docente se enfrenta a un nuevo escenario educativo, en el cual, la mayoría de los jóvenes llevan consigo, dispositivos como lo son el Blackberry, el iPhone, una tablet,

Publicado en formato digital: Lic. Jaquelina E. Escalante, Mgter Sonia I. Mariño y Lic. Romina Y. Alderete . INCORPORANDO HERRAMIENTAS WEB 2.0 EN EL ÁMBITO UNIVERSITARIO. FACEBOOK COMO HERRAMIENTA DE APOYO A LA ENSEÑANZA EN INFORMATICA Revista Geográfica Digital. IGUNNE. Facultad de Humanidades. UNNE. Año 10. No 20. Julio - Diciembre 2013. ISSN 1668-5180 Resistencia, Chaco. 
la laptop, el Smart Phone y un sinnúmero de aparatos que forman parte de las Tecnologías de la Información y la Comunicación( TIC), las cuales forman una parte muy importante en la vida de los jóvenes, por esto representan un área de oportunidad para hacer un intento por mejorar el proceso de enseñanza-aprendizaje en las diversas materias (Naso et. al. 2012).

Las Redes Sociales se pueden definir entre las tecnologías web 2.0 que permiten crear comunidades de práctica en las que participan las personas que comparten ciertos intereses, aficiones y actividades (Panckhurst, 2008) y (Levis, 2010)

El uso de las TIC y la explosión informacional continuamente genera nuevos paradigmas en la institución educativa produciendo grandes cambios respecto a las tecnologías utilizadas y los medios de comunicación. Estas tecnologías Han permitido la integración de servicios de información en el entorno de la enseñanza-aprendizaje, con el fin de dar soporte documental a la actividad docente, de investigación y extensión de la Educación Superior (De Haro, 2010).

El objetivo general de la asignatura Introducción a la Computación es proporcionar una formación sólida en el manejo de los conceptos fundamentales de las TIC y su aplicación en el desempeño laboral del estudiante, fomentando el adecuado empleo e integración de las principales herramientas ofimáticas.

Para lograr aportes significativos, se subraya la importancia de buscar, identificar y solucionar problemas del ámbito profesional y académico. Además, se valora la correcta aplicación de las técnicas y herramientas informáticas específicas.

La Universidad como institución de formación está inmersa en esta sociedad tecnológica donde la información crece de forma rápida, continua y variada, es por eso que tienen la responsabilidad de preparar a las nuevas generaciones, proporcionado conocimientos, habilidades y destrezas que permitirán que los individuos se desarrollen y evolucionen.

Atendiendo a lo expuesto anteriormente se buscó generar una nueva propuesta basada en el uso de las Redes Sociales, en este caso, "facebook", las cuales son parte del diario vivir de los jóvenes, buscando así que el alumno construya su propio aprendizaje y lo relacione de manera particular con sus vivencias, como un recurso didáctico que puede potenciar la enseñanza ofrecida en el aula. Además se considera que el uso de estas herramientas favorecerá notablemente el aprendizaje colaborativo y permitirá a los alumnos desarrollan habilidades comunicativas.

En cuanto a la selección, es importante recordar que "facebook" no es la única red social en línea, existen muchas otras utilizadas por los jóvenes. A partir de 2007, facebook comenzó a desarrollar versiones en español, portugués, francés, alemán y otros idiomas.

Por lo expuesto, se puede constatar la importancia de la relación que lleven a cabo los alumnos, que utilicen las TIC y las herramientas que la ciencia les proporciona, sin ver a la tecnología como un fin, sino como un medio, es decir orientado a generar un aprendizaje significativo.

Entre los años 2009 a 2012 se han detectado algunas problemáticas como ser la desigualdad en saberes previos y la necesidad de transmitir a los ingresantes destrezas requeridas por la sociedad de la información.

Así mismo, la Facultad de Humanidades brinda módulos de Ambientación y Apoyo a fin de promover que los alumnos que ingresan desde el nivel medio se apropien de los mecanismos y modalidades universitarias con miras a disminuir el desgranamiento.

Por lo expuesto, se consideró relevante caracterizar a los alumnos inscriptos y que asisten al modulo opcional, para elaborar propuestas innovadoras, nivelarlos para el cursado de la asignatura en el ciclo

Publicado en formato digital: Lic. Jaquelina E. Escalante, Mgter Sonia I. Mariño y Lic. Romina Y. Alderete . INCORPORANDO HERRAMIENTAS WEB 2.0 EN EL ÁMBITO UNIVERSITARIO. FACEBOOK COMO HERRAMIENTA DE APOYO A LA ENSEÑANZA EN INFORMATICA Revista Geográfica Digital. IGUNNE. Facultad de Humanidades. UNNE. Año 10. № 20. Julio - Diciembre 2013. ISSN 1668-5180 Resistencia, Chaco. 
lectivo y fortalecer los conocimientos de estas herramientas de las TIC e imprescindibles de utilización en la Educación Superior.

\begin{tabular}{|c|c|c|c|c|c|}
\hline Año & $\mathbf{2 0 0 9}$ & $\mathbf{2 0 1 0}$ & $\mathbf{2 0 1 1}$ & $\mathbf{2 0 1 2}$ & $\mathbf{2 0 1 3}$ \\
\hline Ingresantes & 63 & 91 & 88 & 90 & 62 \\
\hline
\end{tabular}

Tabla 1. Ingresantes inscriptos en la asignatura Introducción a la Computación (Fuente: SIU Guaraní Humanidades)

En este trabajo se aborda la caracterización de los alumnos de la asignatura Introducción a la Computación de las carreras Profesorado y Licenciatura en Geografía de la Facultad de Humanidades (UNNE).

Se describen los resultados obtenidos mediante la aplicación de una encuesta a los ingresantes de las carreras Profesorado y Licenciatura en Geografía, donde la información sistematizada sirvió de base para implementar nuevas herramientas informáticas conocidas dentro de la web 2.0 durante el desarrollo de la asignatura, en este caso la implementación de las red social facebook para presentar servicios de comunicación y aprendizaje.

\section{Materiales y Métodos}

La metodología aplicada en este trabajo es de tipo exploratorio y cualitativo. Se basó en las siguientes etapas:

- Selección de destinatarios. El estudio se centró en los estudiantes inscriptos en el año 2013.

- Diseño de instrumentos de relevamiento de datos. Se diseñó una encuesta para caracterizar a los alumnos. Se relevaron dos categorías de datos. Una primera orientada a los datos personales: año de ingreso, género, edad, y si trabaja o no. Una segunda, destinada a relevar datos vinculados a la utilización de Internet y las Redes Sociales en la vida diaria.

- Relevamiento de datos. Se empleó como estrategias para la recolección de datos la encuesta impresa, aplicada al inicio del cuatrimestre en el mes de marzo de 2013.

- Procesamiento y análisis de los datos. Recopilados los datos, se procedió a su sistematización y procesamiento. Se utilizó una herramienta estadística con fines de análisis y evaluación de la situación actual de la temática abordada.

- Implementación y prueba. A partir del relevamiento de la información se procedió a la implementación de una de las Redes Sociales (la más utilizada por los estudiantes, según se detectó en la encuesta), como herramienta colaborativa para apoyar el proceso de enseñanzaaprendizaje.

\section{Resultados}

\section{A- Procesamiento de los datos}

El relevamiento y análisis expuesto generó información preliminar destinada a caracterizar a los alumnos inscriptos en las mencionadas carreras, que cursaron el Modulo de Ambientación y Apoyo en el año 2013.

Publicado en formato digital: Lic. Jaquelina E. Escalante, Mgter Sonia I. Mariño y Lic. Romina Y. Alderete . INCORPORANDO HERRAMIENTAS WEB 2.0 EN EL ÁMBITO UNIVERSITARIO. FACEBOOK COMO HERRAMIENTA DE APOYO A LA ENSEÑANZA EN INFORMATICA Revista Geográfica Digital. IGUNNE. Facultad de Humanidades. UNNE. Año 10. № 20. Julio - Diciembre 2013. ISSN 1668-5180 Resistencia, Chaco. En: http://hum.unne.edu.ar/revistas/geoweb/default.htm 
Se tomó una muestra de 26 alumnos sobre los cuales se aplicó una encuesta para recabar información referente a los aspectos personales, tecnológicos y de formación en TIC con que ingresaban a la facultad, a fin de determinar sus conocimientos y necesidades de contenidos.

A continuación se presentan los resultados obtenidos respecto del: i) Perfil de los alumnos ii) Empleo de las Redes Sociales como herramienta social de las TIC.

\section{i) Perfil de los alumnos}

En esta sección se analizan las características del perfil de los ingresantes. En primer lugar se consideró la edad determinándose que el rango de la mayor parte oscila entre 18-20 años (73\%), luego el 15\% entre $21-23$ años y el restante $12 \%$ supera los 23 años de edad (Figura 1). En la Figura 2 se ilustra el $54 \%$ del género masculino y $46 \%$ del femenino.

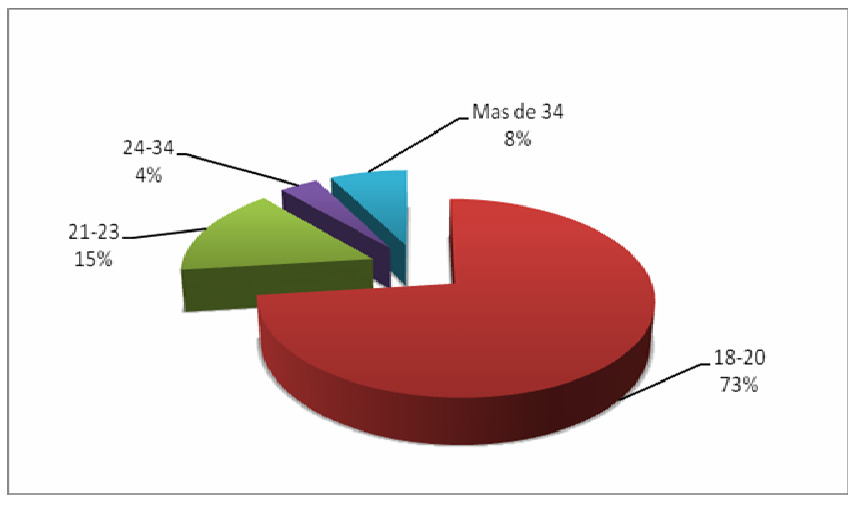

Figura 1. Total de alumnos por edad.

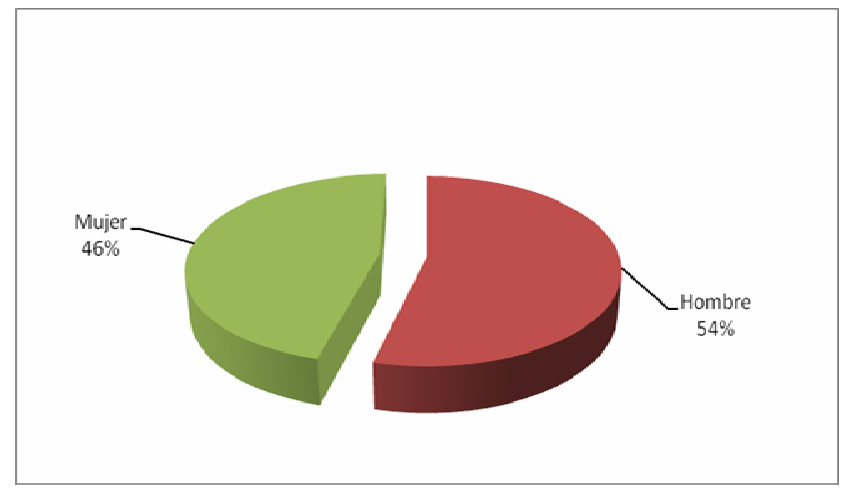

Figura 2.Total de alumnos por género.

Al tratarse de un grupo de estudiantes jóvenes, alrededor del $73 \%$ de los alumnos no supera los 20 años, puede considerarse que influye en los resultados de la siguiente pregunta. El porcentaje de alumnos que no posee ninguna ocupación laboral es del $73 \%$, no obstante un $27 \%$ si trabaja (Figura 3).

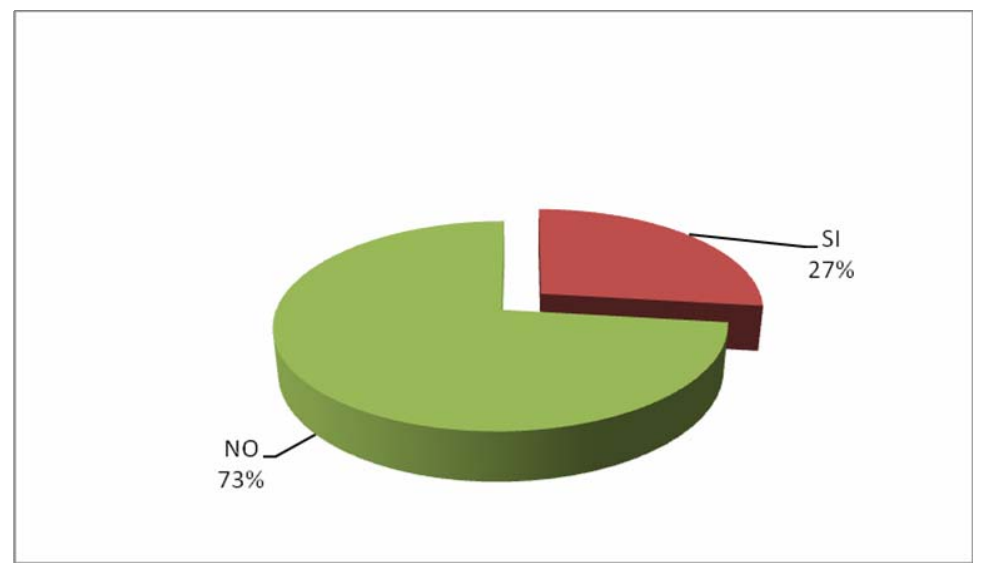

Figura 3. Total de alumnos que trabaja.

ii) Empleo de las Redes Sociales como herramientas de las TIC.

También se consultó a los alumnos respecto a diversos aspectos generales del uso de la tecnología. Se obtuvo información sobre diversos parámetros necesarios para la planificación de actividades en el dictado de la asignatura.

En primera instancia se exploraron que conocimientos poseían los encuestados respecto a las Redes Sociales. Se confirmo que el $100 \%$ de ellos conocen una red social. 
Cuando se indago si usan Redes Sociales el 100\% respondió que afirmativamente, siendo facebook la más utilizada en el $63 \%$ de los casos, seguida en segundo lugar por Twitter con el $17 \%$ y luego las restantes con un menor porcentaje como ser Linkedin, Skype entre otras (Figura 4).

Luego se pregunto respecto al tiempo que utilizan las Redes Sociales, el $27 \%$ respondió que lo utiliza hace aproximadamente 2 años, el 19\% alrededor de un año y el resto de 4 a 5 años de uso (Figura 5). Cabe aclarar que el $88 \%$ cuenta con conexión a Internet en su domicilio particular, mientras que el 12\% restante no dispone de conexión.

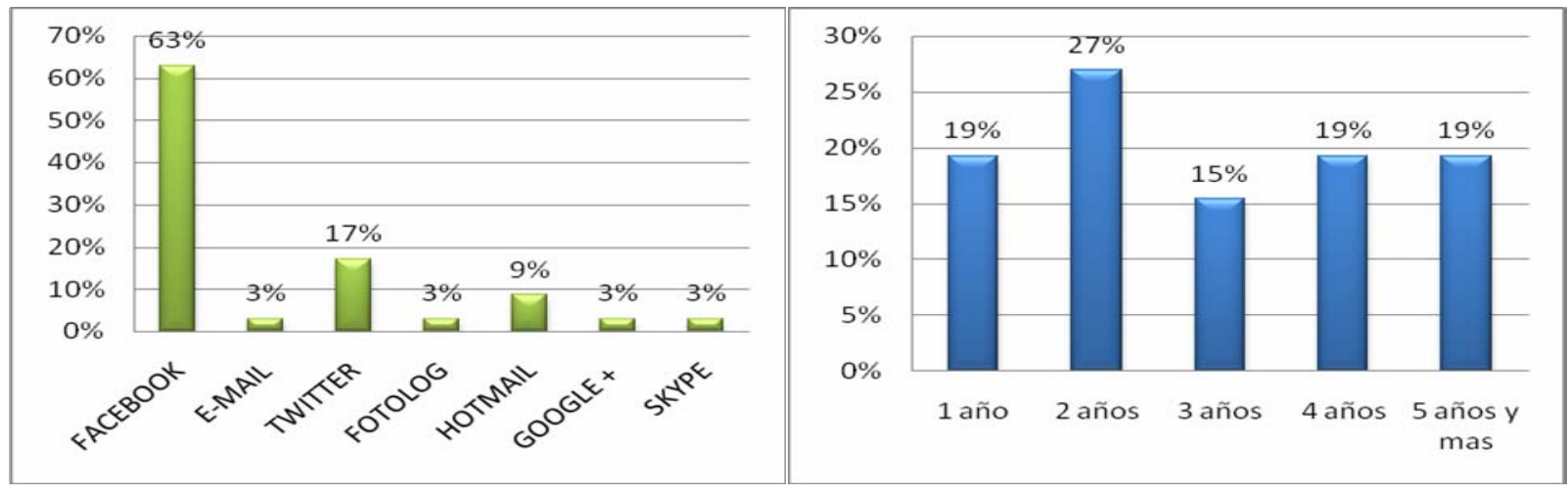

Figura 4. Redes Sociales más utilizadas.

Figura 5. Antigüedad de utilización de las Redes Sociales.

Para determinar la influencia de las Redes Sociales en la vida cotidiana de los alumnos se investigó respecto a la frecuencia de acceso y el tipo de uso de las mismas. En relación a la primera cuestión se vislumbró que el $50 \%$ de los encuestados accede a ellas más de una vez al día, el 35\% ingresa por lo menos una vez al día y el restante $15 \%$ tan solo una vez por semana (Figura 6).

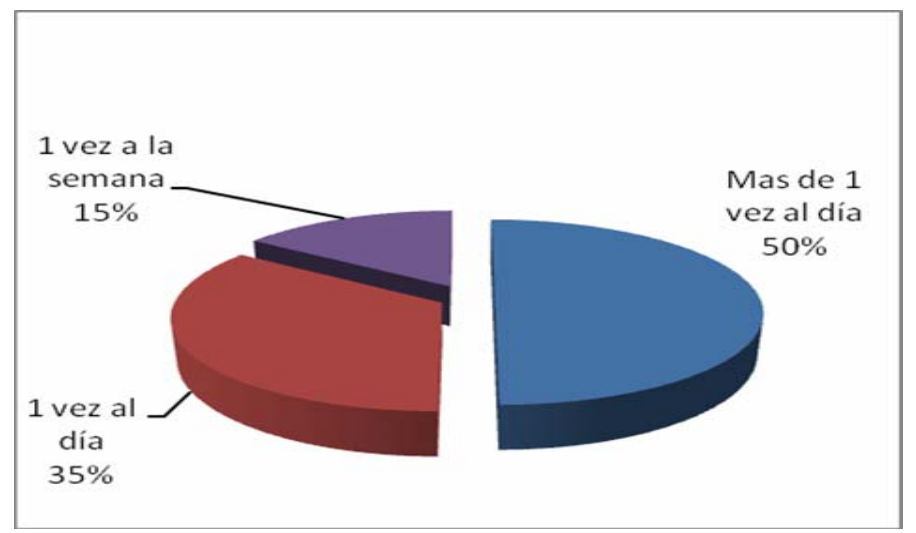

Figura 6. Uso de las Redes Sociales.

\section{B- Implementación de una Red Social en la asignatura.}

Esta asignatura de primer año, es de carácter obligatorio y constituye la única que introduce al alumno en el estudio de las TIC.

Estos alumnos pertenecientes a la generación "nativos digitales", exige a innovar en los métodos de enseñanza-aprendizaje, es por eso que se decidió introducir el uso de una herramienta de la web 2.0

Publicado en formato digital: Lic. Jaquelina E. Escalante, Mgter Sonia I. Mariño y Lic. Romina Y. Alderete . INCORPORANDO HERRAMIENTAS WEB 2.0 EN EL ÁMBITO UNIVERSITARIO. FACEBOOK COMO HERRAMIENTA DE APOYO A LA ENSEÑANZA EN INFORMATICA Revista Geográfica Digital. IGUNNE. Facultad de Humanidades. UNNE. Año 10. No 20. Julio - Diciembre 2013. ISSN 1668-5180 Resistencia, Chaco. 
que permiten el desarrollo de capacidades para trabajar en equipos interdisciplinarios y en forma colaborativa.

En el nuevo escenario de la web 2.0 se seleccionó la red social facebook como herramienta complementaria en el desarrollo de la asignatura. Esta decisión surgió a partir de los datos recabados en las encuestas dado que es la red social más utilizadas por los alumnos.

Se considera que las Redes Sociales son el elemento clave para favorecer este papel activo de los estudiantes. Incorporar el uso de facebook, en la asignatura permitió mostrar otros usos y beneficios de estar conectados a la red. Esto generó una gran ventaja para los alumnos, dado la forma fácil y rápida de promover el diálogo entre pares, se compartieron recursos y se favoreció el aprendizaje.

Desde la asignatura se implementó como herramienta de la web social una cuenta de facebook identificada como "Tutorías Icg UNNE". En la misma se incluyeron los contenidos de los temas desarrollados en las clases presenciales, con el objetivo de proporcionar a los alumnos un mecanismo de tutoría virtual. Básicamente consistió en incorporar a la cuenta Tutorías Icg UNNE, a todos los alumnos inscriptos a cursar en el correspondiente año lectivo, luego a modo de optimizar el seguimiento, se filtro en un grupo de trabajo cerrado, a los que efectivamente cursaron de manera regular las clases de la asignatura (Figura 7).

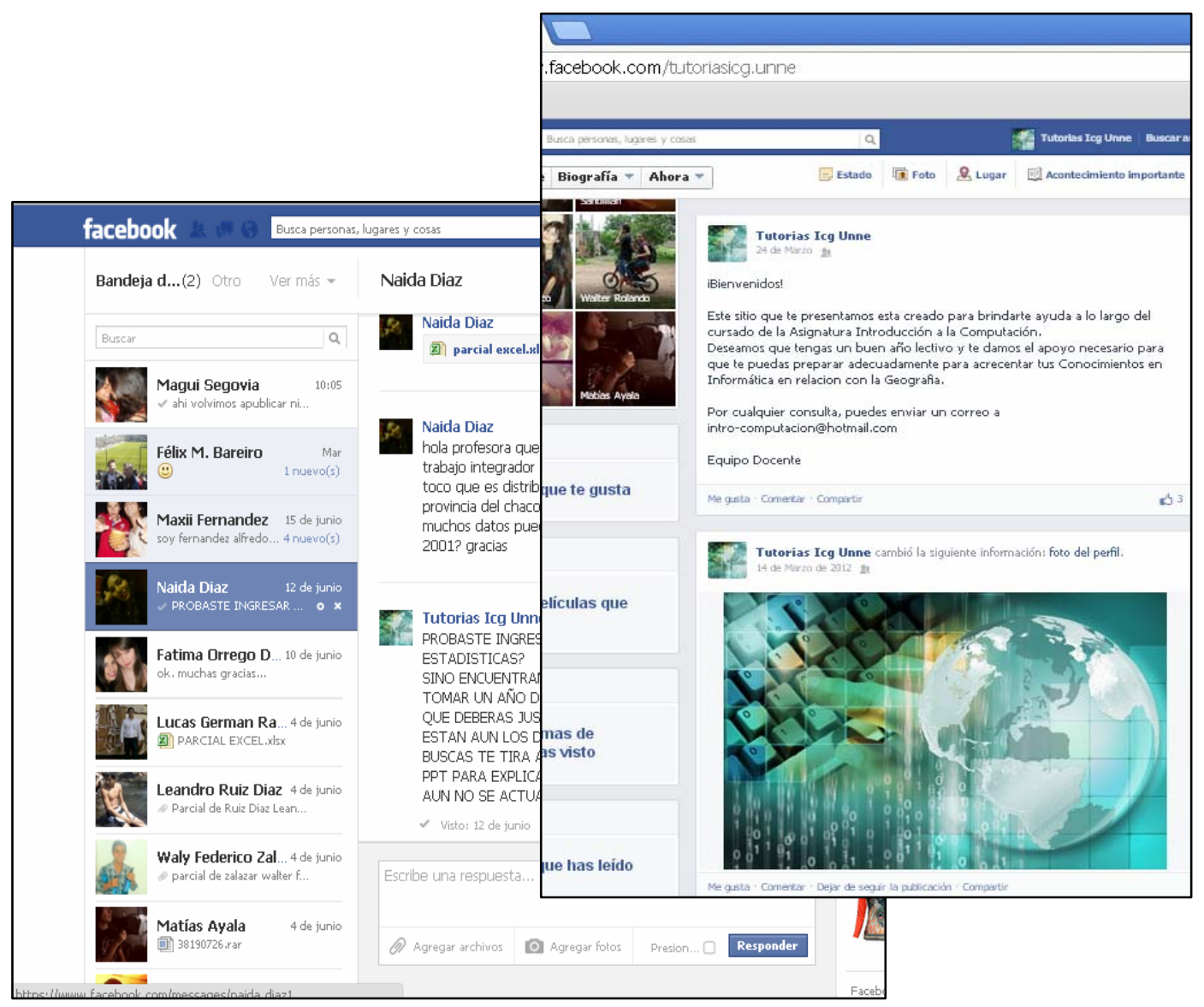

Figura 7. Mensaje de bienvenida y mensajes de consulta

Publicado en formato digital: Lic. Jaquelina E. Escalante, Mgter Sonia I. Mariño y Lic. Romina Y. Alderete . INCORPORANDO HERRAMIENTAS WEB 2.0 EN EL ÁMBITO UNIVERSITARIO. FACEBOOK COMO HERRAMIENTA DE APOYO A LA ENSEÑANZA EN INFORMATICA Revista Geográfica Digital. IGUNNE. Facultad de Humanidades. UNNE. Año 10. № 20. Julio - Diciembre 2013. ISSN 1668-5180 Resistencia, Chaco. En: http://hum.unne.edu.ar/revistas/geoweb/default.htm 
A través del uso de "grupo cerrado", se logró subir los archivos correspondientes a unidades teóricas, trabajos prácticos, y material complementario.

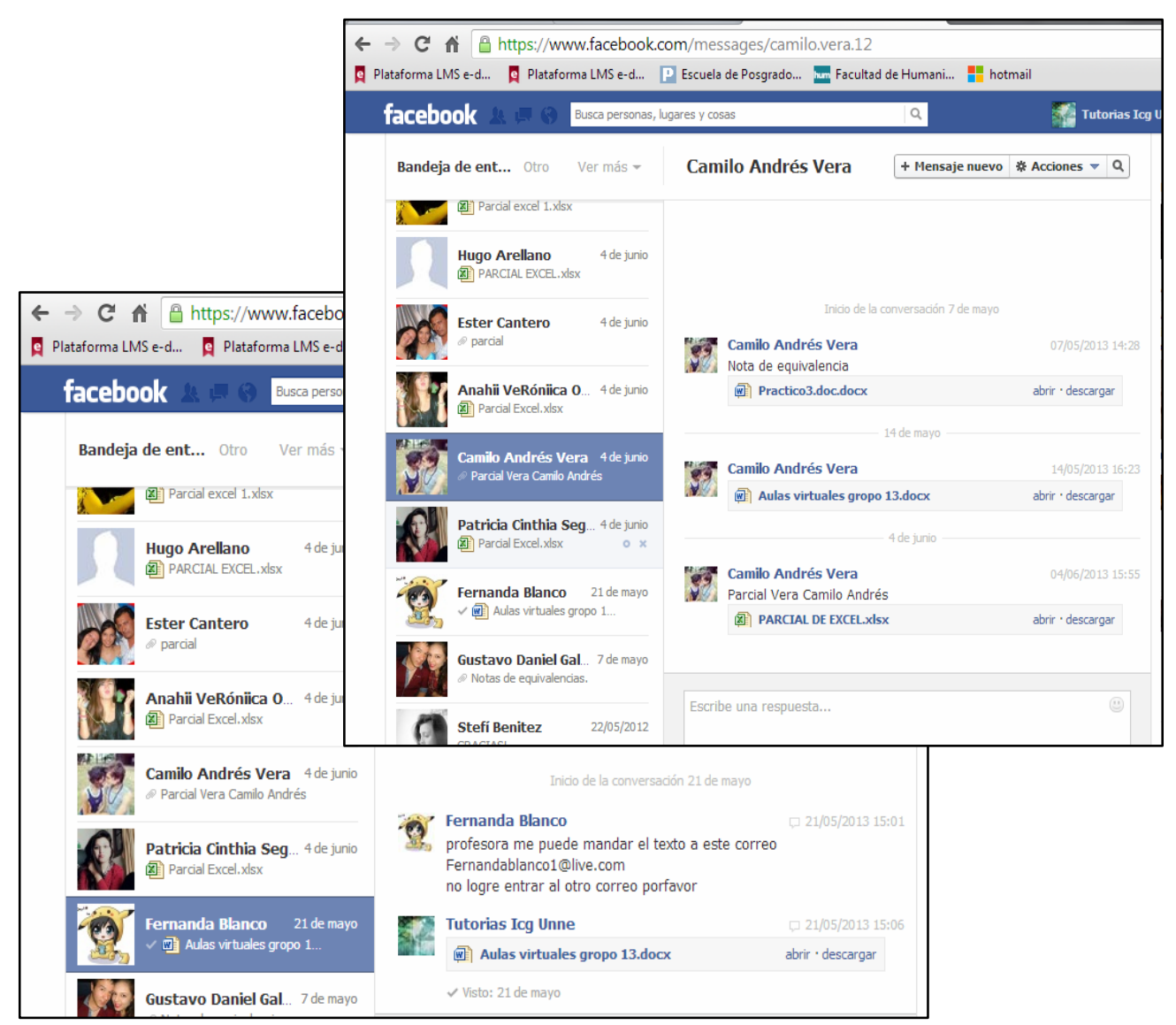

Figura 8. Envío de actividades solicitadas en guías de Trabajos Prácticos

A modo de establecer un diálogo entre docentes y alumnos, se presentaron las consignas prácticas a realizar, posibilitando a los alumnos plantear consultas y dudas relacionadas a los temas abordados. La incorporación a esta red era totalmente voluntaria, ya que el objetivo era realizar una prueba y utilizar esta herramienta como complemento a otras metodologías de enseñanza.

Los resultados fueron muy satisfactorios ya que $100 \%$ de los alumnos adheridos al facebook aprobaron la materia. Además se logró establecer una comunicación más fluida con los alumnos y entre ellos mismos, planteándose casos en donde utilizaban el grupo para difundir información académica entre pares o como intercambio de ideas para ejercicios de trabajos prácticos planteados por la cátedra (Figura 8 y 9).

Se comprobó que su participación en la instancia virtual fue mayor, evidenciándose en los comentarios y consultas realizadas a los docentes de la asignatura.

Publicado en formato digital: Lic. Jaquelina E. Escalante, Mgter Sonia I. Mariño y Lic. Romina Y. Alderete . INCORPORANDO HERRAMIENTAS WEB 2.0 EN EL ÁMBITO UNIVERSITARIO. FACEBOOK COMO HERRAMIENTA DE APOYO A LA ENSEÑANZA EN INFORMATICA Revista Geográfica Digital. IGUNNE. Facultad de Humanidades. UNNE. Año 10. № 20. Julio - Diciembre 2013. ISSN 1668-5180 Resistencia, Chaco. En: http://hum.unne.edu.ar/revistas/geoweb/default.htm 


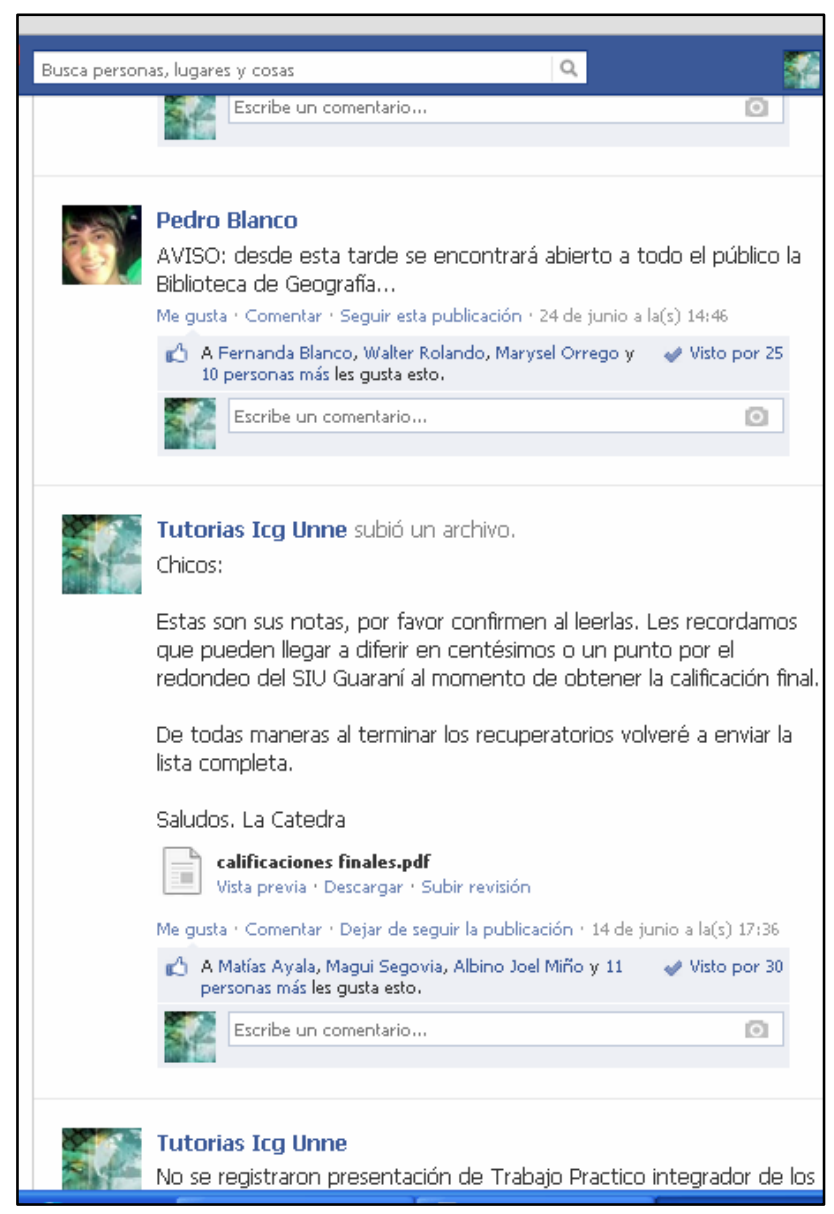

Figura 9. Interacción entre alumnos y profesores

\section{Conclusiones}

En la actualidad, los procesos de enseñanza - aprendizaje han cambiado notablemente y se dirigen hacia un modelo que fomenta la participación del alumno.

Lo innovador del uso de una red social como facebook en la Universidad, se fundamenta en que la incorporación de las TIC favorecen la construcción del aprendizaje significativo

La colaboración y la competencia dentro de dichas herramientas incrementan el aprendizaje al permitirles a los estudiantes trabajar juntos, competir, discutir, asimilar y debatir sobre la veracidad de la información. Por otro lado el proceso de enseñanza- aprendizaje no se limita a una clase o una tarea, sino que se lleva a cabo las 24 horas del día.

Se deducen ventajas como:

- Los alumnos tienen la oportunidad de crear nueva información, retroalimentarse e incrementar el valor de su aportación;

- Dichas tecnologías no son complicadas en su utilización, mucho menos para los nativos digitales;

- Los alumnos cuentan con los recursos necesarios (celulares, tablets, computadoras, conexión a internet);

Publicado en formato digital: Lic. Jaquelina E. Escalante, Mgter Sonia I. Mariño y Lic. Romina Y. Alderete . INCORPORANDO HERRAMIENTAS WEB 2.0 EN EL ÁMBITO UNIVERSITARIO. FACEBOOK COMO HERRAMIENTA DE APOYO A LA ENSEÑANZA EN INFORMATICA Revista Geográfica Digital. IGUNNE. Facultad de Humanidades. UNNE. Año 10. № 20. Julio - Diciembre 2013. ISSN 1668-5180 Resistencia, Chaco. En: http://hum.unne.edu.ar/revistas/geoweb/default.htm 
- La mayoría de los alumnos se encuentran familiarizados con más de una de las mencionadas tecnologías;

- Los estudiantes se convierten en colaboradores en la generación de conocimiento y de información, pues forman parte de la dinámica de aprendizaje;

- Se genera en el estudiante un proceso de análisis y síntesis reforzando su actitud crítica al estar o no estar de acuerdo con otras opiniones disponibles en la herramienta.

Esta web social permitió establecer nuevos vínculos de comunicación entre los docentes y alumnos. Donde los estudiantes se comportaron de manera respetuosa en un mero intercambio informal de información. Además, permitió que los alumnos estén permanentemente al tanto del desarrollo de los contenidos de la asignatura.

A partir del éxito de esta experiencia se prevé su utilización en próximos ciclos incorporando nuevas herramientas de la Web 2.0 que interactúen entre sí a modo de llegar a optimizar un aprendizaje colaborativo entre los cursantes.

Se puso a consideración de los alumnos la posibilidad de implementar la modalidad de trabajo mixta o b-learning o continuar con el dictado sólo presencial de las clases. Asimismo la información relevada y analizada permitirá avanzar en la determinación de las funcionalidades a incluir en una propuesta de plataforma virtual y concretar el aprendizaje mixto o combinado en estos módulos de ambientación y posteriormente en el cursado de la asignatura.

\section{Referencias Citadas}

Naso F., Balbi M. L., Di Grazia N., Peri J. A. 2012. La importancia de las Redes sociales en el ámbito educativo. VII Congreso de Tecnología en Educación y Educación en Tecnología. Junín. Buenos Aires. 2012

De Haro, J. J. 2010. Redes sociales en educación. Ponencia Uso educativo de las redes sociales y participación social de la Jornada Educar para la comunicación y la cooperación social. Universidad de Navarra, mayo 2010.

Fuentes Cotes, M. M. 2013. Diseño del curso de sistemas de gestión ambiental, basado en el modelo pedagógico de aprendizaje significativo, para el mejoramiento continuo del proceso de enseñanza - aprendizaje. Trabajo publicado en el World Engineering Education Forum. (WEEF) 2013. Cartagena Colombia (24 al 27 de Septiembre).

Levis, D. 2011. «Redes educativas 2.1. Medios sociales, entornos colaborativos y procesos de enseñanza y aprendizaje» [artículo en línea]. Revista de Universidad y Sociedad del $\begin{array}{llllll}\text { Conocimiento (RUSC). Vol. } 8, \quad \text { n. } & & 1 & \text { págs. 7-24. UOC. }\end{array}$ http://rusc.uoc.edu/ojs/index.php/rusc/article/view/v8n1-levis/v8n1-levis $\quad$ ISSN 1698-580X (Última consulta 02/05/2013).

McLoughlin, C. \& Lee, M. 2008. Mapping the digital terrain: New media and social software as catalysts for pedagogical change. Proceedings ascilite Melbourne [en línea] Disponible en: http://www.ascilite.org.au/conferences/melbourne08/procs/mcloughlin.html

Santamaría, F. Herramientas colaborativas para la enseñanza usando tecnologías Web: Weblogs, Redes Sociales, Wikis, Web 2.0, octubre 2005.

Rodríguez Navarrete, R.E. 2013. Las redes sociales como estrategia para lograr un aprendizaje significativo de la Historia de México. Revista Congreso Universidad. Vol. II, No. 1, 2013, ISSN: 2306-918X Editorial Universitaria Felix Varela

Valerio, G. \& J. Valenzuela 2010 Competencias informáticas para el e-learning 2.0. Revista Iberoamericana de Educación a Distancia. Disponible en: http://www.utpl.edu.ec/ried/images/pdfs/volumen141/competenciasinformaticas.pdf

i- $\quad$ Twitter: https://twitter.com/

Publicado en formato digital: Lic. Jaquelina E. Escalante, Mgter Sonia I. Mariño y Lic. Romina Y. Alderete . INCORPORANDO HERRAMIENTAS WEB 2.0 EN EL ÁMBITO UNIVERSITARIO. FACEBOOK COMO HERRAMIENTA DE APOYO A LA ENSEÑANZA EN INFORMATICA Revista Geográfica Digital. IGUNNE. Facultad de Humanidades. UNNE. Año 10. № 20. Julio - Diciembre 2013. ISSN 1668-5180 Resistencia, Chaco. 
Revista Geográfica Digital. IGUNNE. Facultad de Humanidades. UNNE. Año 10. № 20. Julio - Diciembre 2013. ISSN 1668-5180 Resistencia, Chaco

ii- Facebook: http://www.facebook.com/

iii- Linkedln: http://www.linkedin.com/

iv- Skype: http://www.skype.com/es/

Publicado en formato digital: Lic. Jaquelina E. Escalante, Mgter Sonia I. Mariño y Lic. Romina Y. Alderete . INCORPORANDO HERRAMIENTAS WEB 2.0 EN EL ÁMBITO UNIVERSITARIO. FACEBOOK COMO HERRAMIENTA DE APOYO A LA ENSEÑANZA EN INFORMATICA Revista Geográfica Digital. IGUNNE. Facultad de Humanidades. UNNE. Año 10. № 20. Julio - Diciembre 2013. ISSN 1668-5180 Resistencia, Chaco. En: http://hum.unne.edu.ar/revistas/geoweb/default.htm 